\title{
Finite State Markov-chain Approximations to Highly Persistent Processes*
}

\author{
Karen A. Kopecky ${ }^{\dagger} \quad$ Richard M. H. Suen ${ }^{\ddagger}$
}

This Version: February 2010

\begin{abstract}
The Rouwenhorst method of approximating stationary AR(1) processes has been overlooked by much of the literature despite having many desirable properties unmatched by other methods. In particular, we prove that it can match the conditional and unconditional mean and variance, and the first-order autocorrelation of any stationary AR(1) process. These properties makes the Rouwenhorst method more reliable than others in approximating highly persistent processes and generating accurate model solutions. To illustrate this, we compare the performances of the Rouwenhorst method and four others in solving the stochastic growth model and an income fluctuation problem. We find that (i) the choice of approximation method can have a large impact on the computed model solutions, and (ii) the Rouwenhorst method is more robust than others with respect to variation in the persistence of the process, the number of points used in the discrete approximation and the procedure used to generate model statistics.
\end{abstract}

Keywords: Numerical Methods, Finite State Approximations

JEL classification: C63.

${ }^{*}$ We thank Russell Cooper, Paul Klein, John Rust, and conference participants at the UCR Conference on Business Cycles, CEF 2009 and the Workshop for Economists Working in Parallel for helpful comments and suggestions. We thank Yundong Tu for excellent research assistance.

${ }^{\dagger}$ Department of Economics, Social Science Center, Room 4701, The University of Western Ontario, London, Ontario, N6A 5C2, Canada. Email: kkopecky@uwo.ca.

${ }^{\ddagger}$ Corresponding author. Department of Economics, Sproul Hall, University of California, Riverside CA 92521-0427. Email: richard.suen@ucr.edu. Tel.: (951) 827-1502. Fax: (951) 827-5685. 


\section{Introduction}

In macroeconomic models, the exogenous stochastic process is typically assumed to follow a stationary first-order autoregressive process. When solving these models numerically, the continuous-valued autoregressive process is usually replaced by a discrete state-space Markov chain. To this end, researchers typically employ the approximation method proposed by Tauchen (1986), or the quadrature-based method developed in Tauchen and Hussey (1991). For AR(1) processes with low persistence, these methods can produce highly accurate approximations. However, their performance deteriorates when the serial correlation is very close to one. ${ }^{1}$ These findings raise concerns because macroeconomic studies typically employ highly persistent processes. In particular, there are two main questions that await answers. First, is there a more reliable technique to approximate highly persistent processes? Second, how does the performance of these methods affect the computed solutions of macroeconomic models? In quantitative studies, approximating the exogenous process is seldom an end in itself. Thus a more appropriate metric for evaluating approximation methods would be their impact on the computed solutions of the entire model. To the best of our knowledge, no existing studies have performed this kind of evaluation. The current study is intended to fill this gap.

Regarding the first question, this paper re-examines a Markov-chain approximation method that is first proposed in Rouwenhorst (1995). The main strength of this method is that it can match five important statistics of any stationary $\mathrm{AR}(1)$ process, including the conditional and unconditional mean, the conditional and unconditional variance, and the first-order autocorrelation. This property makes the Rouwenhorst method more reliable than the other methods in approximating highly persistent processes. The first contribution of this paper is to provide formal proofs of this and other results. ${ }^{2}$

Our second contribution is to compare the performances of five different approximation methods in solving two common macroeconomic models. The methods under study include the Tauchen (1986) method, the original Tauchen-Hussey method, a variation of this method proposed by Flodén (2008a), the Adda-Cooper (2003) method and the Rouwenhorst method. The first model that we consider is the prototypical stochastic neoclassical growth model without leisure. ${ }^{3}$ The main evaluation criterion in

\footnotetext{
${ }^{1}$ This weakness is acknowledged in the original papers. In Tauchen (1986, p.179), the author notes that "Experimentation showed that the quality of the approximation remains good except when $\lambda$ [the serial correlation] is very close to unity." In Tauchen and Hussey (1991), the authors note that for processes with high persistence, "adequate approximation requires successively finer state spaces."

${ }^{2}$ Some of the features of this method are briefly mentioned in Rouwenhorst (1995). But a formal proof of these results is still lacking.

${ }^{3}$ The same model is used in Taylor and Uhlig (1990) and the companion papers to illustrate and compare different solution methods. More recently, Aruoba et al. (2006) use the stochastic growth model, but with labor-leisure choice, to compare different solution methods.
} 
this application is the accuracy in approximating the business cycle moments generated by the model. The second model that we consider is an income fluctuation problem. This problem is of interest because it forms an integral part of the heterogeneous-agent models considered in Aiyagari (1994) and Krusell and Smith (1998). There is now a large literature that uses these models to examine issues in macroeconomics and finance. These models often contain highly persistent processes for individual labor income risk. In some cases, the discretization method used for these processes may be crucial to the validity of the final conclusions. For instance, when these models are used to analyze the welfare implications of policy reforms or eliminating business cycles, the welfare gains or losses are usually quite small. ${ }^{4}$ Thus an accurate approximation could ultimately result in the difference between a welfare gain or loss. When solving the income fluctuation problem, the five methods are evaluated for their accuracy in approximating the degree of inequality in consumption, income and assets. In both models, we use two different approaches to compute the statistics of interest. In the baseline approach the statistics are computed using an approximation to the stationary distribution. In the second approach, the statistics are generated using Monte Carlo simulations.

For the stochastic growth model, regardless of which approach is taken, the choice of approximation method has a large impact on the accuracy of the computed business cycle moments. Moreover, a method that generates a good approximation for the $\mathrm{AR}(1)$ process also tends to yield accurate approximations for the business cycle moments. The Rouwenhorst method has the best performance in this regard. Furthermore, the high degree of accuracy of the Rouwenhorst method prevails even when a coarse state space (with only five states for the exogenous shock) is used. The Tauchen (1986) method has the second best performance, followed by Flodén's variation of the Tauchen-Hussey method. However, these two methods require a much finer state space (at least 25 states) in order to produce the same precision as the Rouwenhorst method. One interesting finding is that the baseline approach, coupled with the Rouwenhorst method, performs as well as the simulation approach.

As for the income fluctuation problem, consistent with our previous findings, the methods which generate good approximations for the $\mathrm{AR}(1)$ process tend to yield more accurate solutions under the baseline approach. The Rouwenhorst method and Flodén's variation have the best performance in this regard. However, the Rouwenhorst method is less sensitive to changes in the number of states in the Markov chain. It is also the only method that produces very similar, yet relatively accurate, results under both the baseline approach and the simulation approach.

\footnotetext{
${ }^{4}$ See, for instance, Krusell et al. (2009) for a recent study that examines the welfare implications of eliminating business cycles in this type of model.
} 
In sum, our quantitative results have two main implications. First, the accuracy of the approximation for the exogenous process can have a large impact on the computed solutions of macroeconomic models. Thus caution must be taken when choosing an approximation method. Second, our results show that the Rouwenhorst method is the most robust of the five methods considered with respect to the degree of persistence of the $\mathrm{AR}(1)$ process, the coarseness of the discrete state space, and the approach used to compute the statistics from the stationary distribution. The accuracies of model solutions computed using the Tauchen (1986) method and the Tauchen-Hussey method, on the other hand, are both sensitive to these choices. It is also worth noting that the performance of the Tauchen (1986) method is extremely sensitive to the choice of a free parameter that determines the bounds on the state space of the discrete process. This feature of the Tauchen (1986) method is overlooked by the existing studies.

The current study is related to Flodén (2008a) and Lkhagvasuren and Galindev (2008). The objective of Flodén (2008a) is to compare the relative performance of various discretization methods in approximating stationary AR(1) processes. However, Flodén does not consider the Rouwenhorst method, nor does he consider the impact of the discretization procedure on the solutions of macroeconomic models. The main objective of Lkhagvasuren and Galindev (2008) is to develop an approximation method for vector autoregressive processes with correlated error terms. These authors show, through a few numerical examples, that the Rouwenhorst method outperforms other methods in approximating moments of univariate $\mathrm{AR}(1)$ processes. In contrast, this result is formally proved in the current study.

\section{The Rouwenhorst Method}

Consider the $\operatorname{AR}(1)$ process

$$
z_{t}=\rho z_{t-1}+\varepsilon_{t}
$$

where $|\rho|<1$ and $\varepsilon_{t}$ is a white noise process with variance $\sigma_{\varepsilon}^{2}$. The $\operatorname{AR}(1)$ process is covariance-stationary with mean zero and variance $\sigma_{z}^{2}=\sigma_{\varepsilon}^{2} /\left(1-\rho^{2}\right)$. If, in addition, $\varepsilon_{t}$ is normally distributed in each period, then $z_{t}$ is also normally distributed. Rouwenhorst (1995) proposes a method to approximate this stochastic process by a discrete state-space process $\left\{y_{t}\right\}$. This involves constructing an $N$-state Markov

chain characterized by (i) a symmetric and evenly-spaced state space $Y_{N}=\left\{\bar{y}_{1}, \ldots, \bar{y}_{N}\right\}$, with $\bar{y}_{1}=-\psi$ and $\bar{y}_{N}=\psi$, and (ii) a transition matrix $\Theta_{N}$. For any $N \geq 2$, the transition matrix $\Theta_{N}$ is determined by two parameters, $p, q \in(0,1)$, and is defined recursively as follows: 
Step 1: When $N=2$, define $\Theta_{2}$ as

$$
\Theta_{2}=\left[\begin{array}{cc}
p & 1-p \\
1-q & q
\end{array}\right]
$$

Step 2: For $N \geq 3$, construct the $N$-by- $N$ matrix

$$
p\left[\begin{array}{cc}
\Theta_{N-1} & \mathbf{0} \\
\mathbf{0}^{\prime} & 0
\end{array}\right]+(1-p)\left[\begin{array}{cc}
\mathbf{0} & \Theta_{N-1} \\
0 & \mathbf{0}^{\prime}
\end{array}\right]+(1-q)\left[\begin{array}{cc}
\mathbf{0}^{\prime} & 0 \\
\Theta_{N-1} & \mathbf{0}
\end{array}\right]+q\left[\begin{array}{cc}
0 & \mathbf{0}^{\prime} \\
\mathbf{0} & \Theta_{N-1}
\end{array}\right],
$$

where $\mathbf{0}$ is a $(N-1)$-by-1 column vector of zeros.

Step 3: Divide all but the top and bottom rows by two so that the elements in each row sum to one.

The main objective of this section is to show formally that the Rouwenhorst method has a number of desirable features unmatched by other methods. However, the matrix $\Theta_{N}$ generated by the procedure above is difficult to work with analytically. Thus, we begin our analysis by offering a new, analytically tractable procedure for generating the Rouwenhorst matrix. The main advantage of this new procedure is that it greatly simplifies the proofs of our analytical results.

\subsection{Reconstructing the Rouwenhorst Matrix}

For any $p, q \in(0,1)$ and for any integer $N \geq 2$, define a system of polynomials as follows

$$
\Phi(t ; N, i) \equiv[p+(1-p) t]^{N-i}(1-q+q t)^{i-1},
$$

for $i=1,2, \ldots, N$. Expanding the polynomials in (2) yields

$$
\Phi(t ; N, i)=\sum_{j=1}^{N} \pi_{i, j}^{(N)} t^{j-1}, \quad \text { for } i=1,2, \ldots, N
$$

Define an $N$-by- $N$ matrix $\Pi_{N}=\left[\pi_{i, j}^{(N)}\right]$ using the coefficients in (3). The main result of this subsection is Proposition 1 which states that the matrix $\Pi_{N}$ is identical to the Rouwenhorst matrix $\Theta_{N}$ for any integer $N \geq 2$. All proofs can be found in the Appendix.

Proposition 1 For any $N \geq 2$ and for any $p, q \in(0,1)$, the matrix $\Pi_{N}$ defined above is identical to the Rouwenhorst matrix $\Theta_{N}$ generated by Steps 1-3. 
Table 1: Selected Moments of the Markov Chain

\begin{tabular}{lll}
\hline \hline Conditional Mean & $\mathrm{E}\left(y_{t+1} \mid y_{t}=\bar{y}_{i}\right)$ & $(q-p) \psi+(p+q-1) \bar{y}_{i}$ \\
Conditional Variance & $\operatorname{var}\left(y_{t+1} \mid y_{t}=\bar{y}_{i}\right)$ & $\frac{4 \psi^{2}}{(N-1)^{2}}[(N-i)(1-p) p+(i-1) q(1-q)]$ \\
Unconditional Mean & $\mathrm{E}\left(y_{t}\right)$ & $\frac{(q-p) \psi}{2-(p+q)}$ \\
Unconditional Second Moment & $\mathrm{E}\left(y_{t}^{2}\right)$ & $\psi^{2}\left\{1-4 s(1-s)+\frac{4 s(1-s)}{N-1}\right\}$ \\
First-order Autocovariance & $\operatorname{Cov}\left(y_{t}, y_{t+1}\right)$ & $(p+q-1) \operatorname{var}\left(y_{t}\right)$ \\
First-order Autocorrelation & $\operatorname{Corr}\left(y_{t}, y_{t+1}\right)$ & $p+q-1$ \\
\hline \hline
\end{tabular}

\subsection{Discrete State-Space Markov Chain}

Consider a Markov chain $\left\{y_{t}\right\}$ with a symmetric and evenly-spaced state space $Y_{N}=\left\{\bar{y}_{1}, \ldots, \bar{y}_{N}\right\}$ defined over the interval $[-\psi, \psi]$. The transition matrix of the Markov chain is given by $\Pi_{N}$, which is a stochastic matrix of non-zero entries. ${ }^{5}$ It follows immediately that the Markov chain has a unique invariant distribution. This result is stated in Proposition 2.

Proposition 2 For any $N \geq 2$, the Markov chain with state space $Y_{N}$ and transition matrix $\Pi_{N}$ has a unique invariant distribution $\boldsymbol{\lambda}^{(N)}=\left(\lambda_{1}^{(N)}, \ldots, \lambda_{N}^{(N)}\right)$, where $\lambda_{i}^{(N)} \geq 0$ and $\sum_{i=1}^{N} \lambda_{i}^{(N)}=1$.

Rouwenhorst mentions that in the symmetric case where $p=q$, the unique invariant distribution is a binomial distribution with parameters $N-1$ and $1 / 2$. Our next result shows that the unique invariant distribution is binomial for any $p, q \in(0,1)$. Since the invariant distribution is unique, it can be solved by the guess-and-verify method. Let $s \equiv \frac{1-q}{2-(p+q)} \in(0,1)$. The guess for $\boldsymbol{\lambda}^{(N)}$, represented by $\hat{\boldsymbol{\lambda}}^{(N)}$, is a binomial distribution with parameters $N-1$ and $1-s$. This means

$$
\widehat{\lambda}_{i}^{(N)}=\left(\begin{array}{c}
N-1 \\
i-1
\end{array}\right) s^{N-i}(1-s)^{i-1}, \quad \text { for } i=1,2, \ldots, N
$$

It is easy to check that this is the actual solution when $N=2$. The result for the general case is established in Proposition 3.

Proposition 3 For any $N \geq 2$, the invariant distribution of the Markov chain defined above is a binomial distribution with parameters $N-1$ and $1-s$.

Equipped with the invariant distribution, one can derive the unconditional moments of the Markov chain. Some of these moments are shown in Table $1 .^{6}$

\footnotetext{
${ }^{5}$ See Lemma 2 in Kopecky and Suen (2009) for a formal proof of this statement.

${ }^{6}$ The mathematical derivations of these results can be found in Kopecky and Suen (2009) Appendix B.
} 


\subsection{Approximating AR(1) Processes}

The task at hand is to approximate a given stationary $\operatorname{AR}(1)$ process with an $N$-state Markov chain. ${ }^{7}$ Let $\left\{z_{t}\right\}$ be the stationary $\operatorname{AR}(1)$ process defined in (1). Conditional on the realization of $z_{t-1}$, the mean and variance of $z_{t}$ are given by $\rho z_{t-1}$ and $\sigma_{\varepsilon}^{2}$, respectively. Now define an $N$-state discrete Markov process $\left\{y_{t}\right\}$ as in Section 2.2 with

$$
p=q=\frac{1+\rho}{2} \quad \text { and } \quad \psi=\sqrt{N-1} \sigma_{z}
$$

Using the equations in Table 1, it is immediate to see that the resulting Markov chain has the same unconditional mean, unconditional variance and first-order autocorrelation as $\left\{z_{t}\right\}$. Suppose $y_{t-1}=\bar{y}_{i}$ for some $\bar{y}_{i}$ in $Y_{N}$. The conditional mean and conditional variance of $y_{t}$ are given by

$$
\mathrm{E}\left(y_{t} \mid y_{t-1}=\bar{y}_{i}\right)=\rho \bar{y}_{i} \quad \text { and } \quad \operatorname{var}\left(y_{t} \mid y_{t-1}=\bar{y}_{i}\right)=\sigma_{\varepsilon}^{2}
$$

Thus $\left\{y_{t}\right\}$ also has the same conditional mean and conditional variance as $\left\{z_{t}\right\}$.

Two remarks regarding this procedure are worth mentioning. First, under the Rouwenhorst method, the approximate Markov chain is constructed using $\rho$ and $\sigma_{\varepsilon}^{2}$ alone. In particular, the transition matrix $\Pi_{N}$ is not a discretized version of the conditional distribution of $z_{t}$. This is the fundamental difference between this method and the ones proposed in Tauchen (1986) and Tauchen and Hussey (1991). Second, the above procedure can be applied to any stationary $\mathrm{AR}(1)$ process, including those with very high persistence. Thus, unlike the other two methods, the one proposed by Rouwenhorst can always match the unconditional variance and the persistence of $\left\{z_{t}\right\}$.

Since the invariant distribution of $\left\{y_{t}\right\}$ is a binomial distribution with mean zero and variance $\sigma_{y}^{2}=$ $\sigma_{\varepsilon}^{2} /\left(1-\rho^{2}\right)$, the standardized process $\left\{y_{t} / \sigma_{y}\right\}$ converges to the standard normal distribution as $N$ goes to infinity. Thus the Rouwenhorst method is particularly apt for approximating Gaussian AR(1) processes.

\section{Evaluations}

In this section we examine the performance of the Rouwenhorst method and four other discretization methods in solving the stochastic growth model and the income fluctuation problem. For the stochastic

\footnotetext{
${ }^{7}$ In this paper, we focus on univariate $\operatorname{AR}(1)$ processes only. For vector autoregressive processes, one can combine the Rouwenhorst method with the decomposition method proposed in Lkhagvasuren and Galindev (2008). More specifically, these authors propose a method to decompose a multivariate process into a number of independent univariate processes. These independent processes can then be approximated using the Rouwenhorst method described below.
} 
growth model, the main evaluation criterion is the accuracy in approximating the business cycle moments generated by the model. For the income fluctuation problem, we focus on measures of inequality in consumption, income and assets. The other methods under evaluation are described below.

Tauchen (1986) method Under this method, an evenly-spaced state space $Y_{N}=\left\{\bar{y}_{1}, \ldots, \bar{y}_{N}\right\}$ is used to construct the Markov chain $\left\{y_{t}\right\}$, with $\bar{y}_{N}=-\bar{y}_{1}=\Omega \sigma_{z}$, where $\Omega$ is a positive real number and $\sigma_{z}$ is the standard deviation of the original $\operatorname{AR}(1)$ process. Let $\Phi$ be the probability distribution function for the standard normal distribution. For any $i=1, \ldots, N$, the transition probabilities of the Markov chain are given by

$$
\pi_{i, j}=\Phi\left(\frac{\bar{y}_{j}-\rho \bar{y}_{i}+h / 2}{\sigma_{\varepsilon}}\right)
$$

for $j=1$ and $N$, and

$$
\pi_{i, j}=\Phi\left(\frac{\bar{y}_{j}-\rho \bar{y}_{i}+h / 2}{\sigma_{\varepsilon}}\right)-\Phi\left(\frac{\bar{y}_{j}-\rho \bar{y}_{i}-h / 2}{\sigma_{\varepsilon}}\right)
$$

for $j=2, \ldots, N-1$, where $h$ is the step size between the grid points. It turns out that the performance of this method is strongly affected by the choice of $\Omega$. To the best of our knowledge, there is no established rule for determining this parameter. ${ }^{8}$ In all the results reported below, $\Omega$ is calibrated such that the standard deviation of $\left\{y_{t}\right\}$ matches the standard deviation of the $\mathrm{AR}(1)$ process. This gives the method its best chance in approximating the $\mathrm{AR}(1)$ process. $^{9}$

The Quadrature-Based Methods Under this class of methods, the elements of the state space are determined by $\bar{y}_{i}=\sqrt{2} \sigma x_{i}$, for $i=1,2, \ldots, N$, where $\left\{x_{i}\right\}$ are the Gauss-Hermite nodes defined on $[-\infty, \infty]$. Let $\left\{\phi_{j}\right\}$ be the corresponding Gauss-Hermite weights. The elements in the transition matrix $\Pi$ are then given by

$$
\pi_{i, j}=\frac{f\left(\bar{y}_{j} \mid \bar{y}_{i}\right)}{f\left(\bar{y}_{j} \mid 0\right)} \frac{\bar{w}_{j}}{s_{i}}
$$

where $\bar{w}_{j}=\phi_{j} / \sqrt{\pi}$, the function $f\left(\cdot \mid \bar{y}_{i}\right)$ is the density function for $N\left(\rho \bar{y}_{i}, \sigma^{2}\right)$, and

$$
s_{i}=\sum_{n=1}^{N} \frac{f\left(\bar{y}_{n} \mid \bar{y}_{i}\right)}{f\left(\bar{y}_{n} \mid 0\right)} \bar{w}_{n} .
$$

\footnotetext{
${ }^{8}$ Tauchen (1986) sets $\Omega=3$ without giving any justification. Flodén (2008) sets $\Omega=1.2 \ln (N)$. As explained in Section 3.1, Flodén's choice of $\Omega$ is the main reason why he finds that the Tauchen (1986) method performs poorly in approximating highly persistent processes.

${ }^{9}$ We choose to target $\sigma_{z}$ instead of $\rho$ because, relative to $\sigma_{z}$, the persistence parameter $\rho$ is well approximated under this method for a range of values of $\Omega$ and degrees of persistence.
} 
In Tauchen and Hussey (1991), the standard deviation $\sigma$ is taken to be $\sigma_{\varepsilon}$. In Flodén (2008a), $\sigma$ is a weighted average of $\sigma_{z}$ and $\sigma_{\varepsilon}$. In particular, $\sigma=\omega \sigma_{\varepsilon}+(1-\omega) \sigma_{z}$ with $\omega=0.5+0.25 \rho$.

The Adda-Cooper (2003) Method The first step of this method is to partition the real line into $N$ intervals. Formally, let $I_{n}=\left[x_{n}, x_{n+1}\right]$ be the $n$th interval with $x_{1}=-\infty$ and $x_{N+1}=+\infty$. The cut-off points $\left\{x_{n}\right\}_{n=2}^{N}$ are the solutions of the following system of equations:

$$
\Phi\left(\frac{x_{n+1}}{\sigma_{z}}\right)-\Phi\left(\frac{x_{n}}{\sigma_{z}}\right)=\frac{1}{N}, \quad \text { for } n=1,2, \ldots, N
$$

where $\Phi$ is the probability distribution function for the standard normal distribution. The $n$th element in the state space is the mean value of the $n$th interval. For any $i, j \in\{1,2, \ldots, N\}$, the transition probability $\pi_{i, j}$ is defined as the probability of moving from interval $I_{i}$ to interval $I_{j}$ in one period.

\subsection{Stochastic Growth Model}

Consider the planner's problem in the stochastic growth model,

$$
\max _{\left\{C_{t}, K_{t+1}\right\}_{t=0}^{\infty}} E_{0}\left[\sum_{t=0}^{\infty} \beta^{t} \log \left(C_{t}\right)\right]
$$

subject to

$$
\begin{aligned}
& C_{t}+K_{t+1}=\exp \left(a_{t}\right) K_{t}^{\alpha}+(1-\delta) K_{t}, \\
& a_{t+1}=\rho a_{t}+\varepsilon_{t+1}, \quad \text { with } \rho \in(0,1),
\end{aligned}
$$

$C_{t}, K_{t+1} \geq 0$, and $K_{0}$ given, where $C_{t}$ denotes consumption at time $t, K_{t}$ denotes capital, $A_{t} \equiv \exp \left(a_{t}\right)$ is the technological factor and $\varepsilon_{t+1} \sim$ i.i.d. $N\left(0, \sigma_{\varepsilon}^{2}\right)$. The parameter $\beta \in(0,1)$ is the subjective discount factor, $\alpha \in(0,1)$ is the share of capital income in total output and $\delta \in(0,1]$ is the depreciation rate.

The Bellman equation for this problem is

$$
V(K, a)=\max _{K^{\prime}}\left\{\log \left(\exp (a) K^{\alpha}+(1-\delta) K-K^{\prime}\right)+\beta \int V\left(K^{\prime}, a^{\prime}\right) d F\left(a^{\prime} \mid a\right)\right\}
$$

where $F(\cdot \mid a)$ is the distribution function of $a_{t+1}$ conditional on $a_{t}=a$. 


\section{Parameterization and Computation}

Following King and Rebelo (1999), we use the following parameter values: $\alpha=0.33, \beta=0.984, \delta=0.025$, $\sigma_{\varepsilon}=0.0072$ and $\rho=0.979$. Under this parameterization, the business cycle moments of interest do not have closed-form solutions. Thus we first compute a highly accurate approximation of these moments. To do this we use the Chebyshev parameterized expectation algorithm described in Christiano and Fisher (2000) to compute the policy function. ${ }^{10}$ We then generate a sequence of $a_{t}$ of length 50,010,000 using the actual AR(1) process. The first 10,000 observations are discarded (the "burn-in") and the rest are used to compute the business cycle moments. The solutions obtained will be referred to as the "quasi-exact" solutions of the model.

We then compute the business cycle moments using the discretization methods mentioned above. First, the $\operatorname{AR}(1)$ process in (6) is replaced by a Markov chain with state space $\mathcal{A}=\left\{\bar{a}_{1}, \ldots, \bar{a}_{N}\right\}$ and transition matrix $\Pi=\left[\pi_{i, j}\right]$. Next, we form an evenly-spaced grid for $k \equiv \ln K$, represented by $\mathcal{K}=$ $\left\{\bar{k}_{1}, \ldots, \bar{k}_{M}\right\}$. In the results reported below, we set $M=1,000$ and use three different values for $N$, namely 5, 10 and 25. The Bellman equation in (7) is then solved over the discrete state space $\widehat{\mathcal{S}}=\mathcal{K} \times \mathcal{A}$ using the value-function iteration method described in Tauchen (1990) and Burnside (1999). The outcome is a discrete approximation to the policy function, denoted by $\left\{\widehat{g}\left(\bar{k}_{m}, \bar{a}_{n}\right):\left(\bar{k}_{m}, \bar{a}_{n}\right) \in \widehat{\mathcal{S}}\right\}$.

The business cycle moments are then computed using two different approaches. Under the baseline approach, an approximation to the stationary distribution of the state variables $(k, a)$ is computed by iterating on the equation

$$
\widetilde{\pi}^{l} P=\widetilde{\pi}^{l+1}
$$

where $P$ is the transition matrix for $(k, a)$. The iterations proceed until the "distance" between successive

iterates, as measured by $\max \left|\widetilde{\boldsymbol{\pi}}^{l}-\widetilde{\boldsymbol{\pi}}^{l+1}\right|$, is within the desired tolerance. The business cycle moments are then derived using $\widetilde{\boldsymbol{\pi}}^{l}$ and the computed policy function $\widehat{g}$. Under the second approach, the business cycle moments are generated using Monte Carlo simulations. First, we generate a common sequence of $a_{t}$ of length 5,010,000 using the actual $\mathrm{AR}(1)$ process with a burn-in period of 10,000. We then use the computed policy function to construct a sequence of $k_{t} \equiv \ln K_{t}$. Linear interpolation is used to compute

\footnotetext{
${ }^{10}$ Specifically, we compute the continuous shock version of the model using the Chebyshev parameterized expectation approach and the Wright-William specification of the conditional expectation function. The conditional expectation function is approximated by $\sum_{i=1}^{N} \theta_{i} C_{i}(k, a)$, where $C_{i}(k, a), i=1, \ldots, N$ are the elements of the set $\left\{T_{i 1}(\phi(k)) T_{i 2}(\psi(a)) \mid \sum_{j=1}^{2} i_{j} \leq\right.$ $n\}$ and $\theta_{i}, i=1, \ldots, N$ are the weights. The functions $T_{i j}$ for $j=1,2$ are the $i$ th Chebyshev polynomials and $\phi$ and $\psi$ are linear mappings of $\left[k_{\min }, k_{\max }\right]$ and $[-3 \sigma, 3 \sigma]$ into the interval $[-1,1]$. We set $n=12$ so that $N=78$ and we use $M=2,916$ quadrature nodes, 54 in each direction. Further increasing $N$ and $M$ results in a less than 1 percent change in all the business cycle moments computed. Following Christiano and Fisher (2000), the conditional expectation is computed using 4-point Gauss-Hermite quadrature.
} 
values of $\widehat{g}\left(k_{t}, a_{t}\right)$ for points not in the discrete state space $\widehat{\mathcal{S}}$.

One major difference between these two approaches is the sources of the errors that they introduce. While both methods suffer from errors in the computation of the policy function, under the baseline approach, additional errors occur due to the discrete approximation of the stationary distribution. However, this approach does not suffer from the approximation errors due to linear interpolation and the sampling errors generated by the simulation method. ${ }^{11}$

\section{Results}

Panel (A) of Table 2 shows the ratio of the computed statistics obtained under the baseline approach to the quasi-exact solutions. ${ }^{12}$ First, we consider the performance of the five methods in approximating the original $\mathrm{AR}(1)$ process. As explained in Section 2.3, the parameters in the Rouwenhorst method can be calibrated to match exactly the moments $\left\{\rho, \sigma_{\varepsilon}, \sigma_{a}\right\}$. Similarly, the parameter $\Omega$ in the Tauchen (1986) method is calibrated to match the standard deviation of $a_{t}$. Under this procedure, the Tauchen (1986) method yields a very small relative error (less than one percent) in approximating $\rho$ under all three values of $N$. Our results are in stark contrast to those reported in Flodén (2008a) Table 2. Using $\Omega=1.9313$ and $N=5$, Flodén reports that this method produces a 12 percent error in approximating $\sigma_{a}$ and 1.5 percent error in approximating $\rho$. This illustrates that the performance of the Tauchen (1986) method is very sensitive to the choice of $\Omega$.

In general, a discretization method that generates an accurate approximation for $\rho$ and $\sigma_{a}$ also has high precision in approximating the business cycle moments. The Rouwenhorst method has the best overall performance in this regard. Moreover, this method is capable of producing highly accurate approximations even when $N$ is small. The Tauchen (1986) method has the second best overall performance, followed by Flodén's variation of the Tauchen-Hussey method. However, the performances of these two methods are extremely sensitive to the size of the grid for $a_{t}$ and deteriorate significantly when $N$ decreases. Finally, it is worth noting that the five methods have very different performances in approximating the covariance between $k_{t}$ and $a_{t}$, especially when $N$ is small. A method that generates a good approximation for this statistic also tends to yield accurate approximations for the covariances

\footnotetext{
${ }^{11}$ Santo and Peralta-Alva (2005) show that, under some mild conditions, the moments of the endogenous variables generated from Monte Carlo simulations will converge to the exact values as the sample size approaches infinity. In practice, we can only use finite samples and so some sampling errors remain. These errors, however, are small relative to the errors caused by linear interpolation.

${ }^{12}$ The reported statistics include the moments of the actual $\operatorname{AR}(1)$ process, $\left\{\rho, \sigma_{\varepsilon}, \sigma_{a}\right\}$, the standard deviations of capital, output, consumption and investment (all in logarithmic terms), the covariance between $k_{t}$ and $a_{t}$, and the firstorder autocorrelation of output (in logarithmic terms). The first-order autocorrelations of other variables, and the crosscorrelations between output and other variables are not shown in the paper but are available from the authors upon request.
} 
between $y_{t}$ and other endogenous variables. It is thus important to choose a method that can match this statistic well. As the table shows, the Rouwenhorst method generates the most accurate approximation of this covariance and, as a result, the rest of the business cycle moments. ${ }^{13}$

Panel (B) of Table 2 reports the simulation results. These results show that the choice of discretization method matters even when the business cycle moments are computed using Monte Carlo simulations. This is, in part, because linear interpolation is used to approximate $g\left(k_{t}, a_{t}\right)$ for values of $k_{t}$ and $a_{t}$ that are outside the discrete state space. The size of the error due to the interpolation procedure depends on the location of the grid points and hence the choice of the discretization method. However, as $N$ increases, the state space becomes finer and the overall error due to interpolation decreases. For the Rouwenhorst method, a five-fold increase in $N$ only marginally affects its precision. In fact, this method is able to produce highly accurate approximations even when $N=5$. But for the other methods, such an increase in $N$ generates a significant improvement in their performance. Consequently, it is only with 25 states in the Markov chain that the Tauchen (1986) method, the Tauchen-Hussey method and Flodén's variation can achieve degrees of accuracy on par with the Rouwenhorst method.

Two additional observations of Table 2 are worth noting. First, in terms of solving the stochastic growth model, value-function iteration, together with a five-state Markov chain constructed using the Rouwenhorst method, produces highly accurate results that are nearly identical to the quasi-exact solutions computed using Chebyshev PEA. This is an important finding because the first method is significantly easier to implement and requires substantially less computational time than the latter (32 seconds versus 65.25 minutes). ${ }^{14}$ Second, when comparing between the two panels of Table 2, one can see that the baseline approach, when combined with the Rouwenhorst method, can generate estimated moments that are as accurate as those produced by the simulation method with five million draws. Our results thus show that simulation is not necessary to generate accurate statistics. In fact, it may result in less accuracy than the baseline approach if the sample size is too small. ${ }^{15}$

\subsection{Income Fluctuation Problem}

Consider an infinitely-lived, risk-averse consumer who receives a random labor endowment $e_{t}$ in each period $t$. The agent can self-insure by borrowing and lending via a single risk-free asset but there is an

\footnotetext{
${ }^{13}$ These results are not shown in the paper to conserve space but are available from the authors upon request.

${ }^{14}$ This is the amount of time that the value-function iteration method takes given an initial guess of 0 and that the Chebyshev PEA takes given an initial guess that is fairly close to the actual solution. For the Chebyshev PEA, about $2 / 3$ of the run-time is spent generating the 50,010,000 draws from the exogenous shock process.

${ }^{15}$ For instance, the baseline approach with the Rouwenhorst method yields more accurate statistics than the simulation method when only one million draws are used.
} 
upper bound on how much he can borrow. Formally, the consumer's problem is given by

$$
\max _{\left\{c_{t}, a_{t+1}\right\}_{t=0}^{\infty}} E_{0}\left[\sum_{t=0}^{\infty} \beta^{t} \log \left(c_{t}\right)\right]
$$

subject to

$$
\begin{gathered}
c_{t}+a_{t+1}=w e_{t}+(1+r) a_{t}, \\
\ln e_{t+1}=\rho \ln e_{t}+\varepsilon_{t+1}, \quad \text { with } \rho \in(0,1),
\end{gathered}
$$

$c_{t} \geq 0$, and $a_{t+1} \geq-\underline{a}$. The variable $c_{t}$ denotes period $t$ consumption, $a_{t}$ denotes period $t$ assets, $w$ is the wage, $r$ is the return on assets, $\underline{a} \geq 0$ is the borrowing limit and $\varepsilon_{t+1} \sim$ i.i.d. $N\left(0, \sigma_{\varepsilon}^{2}\right)$.

The Bellman equation for the consumer's problem is given by

$$
V(a, e)=\max _{a^{\prime}}\left\{\log \left(w e+(1+r) a-a^{\prime}\right)+\beta \int V\left(a^{\prime}, e^{\prime}\right) d F\left(e^{\prime} \mid e\right)\right\}
$$

where $F(\cdot \mid e)$ is the distribution function of $e_{t+1}$ conditional on $e_{t}=e$.

\section{Parameterization and Computation}

The following parameter values are used in the computation. The subjective discount factor $\beta$ is chosen to be 0.96 . The borrowing limit $\underline{a}$ is set to zero so that no borrowing is allowed. The rate of return $r$ is taken to be 3.75 percent and the wage rate is normalized to one. As for the labor endowment process, we consider two different specifications that are commonly used in the literature. In the first specification, we follow Aiyagari (1994) and set $\rho=0.90$ and $\sigma_{\varepsilon}=0.2$. In the second specification, we use the estimates obtained by French (2005), which are $\rho=0.977$ and $\sigma_{\varepsilon}=0.12 .^{16}$

The computational procedure is similar to the one described in Section 3.1. First, we compute a highly accurate approximation of the inequality measures of interest. Again we use the Chebyshev PEA to compute the policy function $a^{\prime}=g(a, e) \cdot{ }^{17}$ We then generate a sequence of $\ln e_{t}$ of length 50,010,000 using the actual $\mathrm{AR}(1)$ process with a burn-in period of 10,000. We then use this and the computed policy function to construct two inequality measures for consumption, total income and assets. These measures are the coefficient of variation (CV) and the Gini coefficient. Next, we use value-function iteration with

\footnotetext{
${ }^{16}$ Storesletten et al. (1999) report similar estimates for $\rho$ and $\sigma_{\varepsilon}$. Pijoan-Mas (2006) uses the estimates reported in French (2005) in his calibration. Similar values for $\rho$ and $\sigma_{\varepsilon}$ are used in other studies including Chang and Kim (2006, 2007) and Flodén (2008b).

${ }^{17}$ Specifically, the same method described in footnote 10 is used to approximate the conditional expectation function. The only difference is, in this case, we set $n=23$ so that $N=276$ and we use $M=40,000$, with 200 nodes in each direction. Further increasing $N$ and $M$ results in a less than 1 percent change in all the moments computed.
} 
linear interpolation on the value function to solve the Bellman equation in (9) on a discrete state space $\widehat{\mathcal{S}}$. Specifically, we form a 25-state Markov chain using each of the five methods and use 1,500 grid points for assets. ${ }^{18}$ We then compute the two inequality measures using the baseline approach and Monte Carlo simulations. In the baseline approach, we use 15,000 grid points for assets to compute the stationary distribution. In the Monte Carlo simulations, we generate a sequence of $\ln e_{t}$ of length 5,010,000 using the actual $\mathrm{AR}(1)$ process with a burn-in period of 10,000. The sequence so obtained is used to compute the inequality measures.

\section{Results}

The ratios of the inequality measures obtained under the baseline approach and the simulation approach to the quasi-exact solutions are shown in Panels (A) and (B) of Table 3. The table shows that for some inequality measures, the results obtained from the discrete state space method differ significantly from the quasi-exact solutions. In particular, for all five discretization methods considered, the discrete state space method tends to underestimate the degree of wealth inequality under both approaches. This problem remains even when a 25-state grid for $e_{t}$ is used and arises from errors in the approximation of the policy function that occur when the domain for $e_{t}$ is discretized. ${ }^{19}$

The table also shows that the choice of discretization method is important when using the baseline approach. Moreover, under this approach, methods that generate relatively more accurate approximations for the persistence and the standard deviation of the $\mathrm{AR}(1)$ process also tend to yield relatively more accurate solutions. This is consistent with the findings for the stochastic growth model. Under Aiyagari's specification of the labor endowment process, and with $N=25$, the Tauchen-Hussey method, Flodén's variation and the Rouwenhorst method have the best performance. Under French's specification, where the $\mathrm{AR}(1)$ process is more persistent, Flodén's variation and the Rouwenhorst method continue to have the best performance but the accuracy of the Tauchen-Hussey method deteriorates significantly. Thus Flodén's variation and the Rouwenhorst method are more robust to variations in $\rho$. However, the performance of Flodén's method is rather sensitive to the choice of $N$. In particular, the accuracy of this method decreases considerably when $N$ is lowered from 25 to 10 . Meanwhile, the accuracy of the Rouwenhorst method is only marginally affected by this change. These findings illustrate that, under this approach, only the Rouwenhorst method is robust to changes in both $N$ and $\rho$.

\footnotetext{
${ }^{18}$ We use a transformation of assets so that there are more grid points around the borrowing limit $\underline{a}$. The resulting grid points are thus not evenly spaced. This procedure is commonly used in solving the income fluctuation problem. See, for instance, den Haan (2010).

${ }^{19}$ Note that this result is not due to the coarseness of the asset grids as doubling their size does not improve the accuracies of these statistics.
} 
In contrast, under the simulation approach, all five methods yield very similar results when Aiyagari's specification is used. When $\rho$ is increased to 0.977 , larger differences in the simulation results are observed. However, in this case, no single method dominates the others in all measures. These results show that a significant amount of the variation in accuracy of the different methods under the baseline approach is due to variation in the accuracy of the discrete approximation to the stationary distribution. Comparing across the two approaches, note that while some methods perform better than the Rouwenhorst method in some cases, the Rouwenhorst method is the most consistent across the two approaches.

In sum, the choice of discretization method can have a significant impact on the accuracy of model solutions. The Rouwenhorst method is found to be one of the most accurate among the five methods considered. Moreover, it is the most robust to variations in the persistence of the exogenous process, the number of states in the Markov chain, and the approach used in obtaining the statistics from the stationary distribution.

\section{Conclusions}

The main contributions of this paper are two-fold. First, it re-examines the Rouwenhorst method of approximating stationary $\mathrm{AR}(1)$ processes and shows formally that this method can match five important statistics of any stationary $\mathrm{AR}(1)$ process. This property makes the Rouwenhorst method more reliable than other methods in approximating highly persistent processes. Second, it compares the performances of the Rouwenhorst method and four other methods in solving the stochastic growth model and a standard income fluctuation problem. Our quantitative results show that the accuracy of the approximation for the exogenous process can have a large impact on the computed solutions of these models. In particular, a good approximation for the persistence and the standard deviation of the AR(1) process is important for obtaining accurate approximations of statistics generated from the models. The Rouwenhorst method has one of the best performances in these regards. This is because, unlike the other methods, it can generate relatively accurate solutions when the persistence of the exogenous process is very close to one regardless of the coarseness of the state space for the Markov chain or the approach used to compute the statistics from the stationary distribution. 


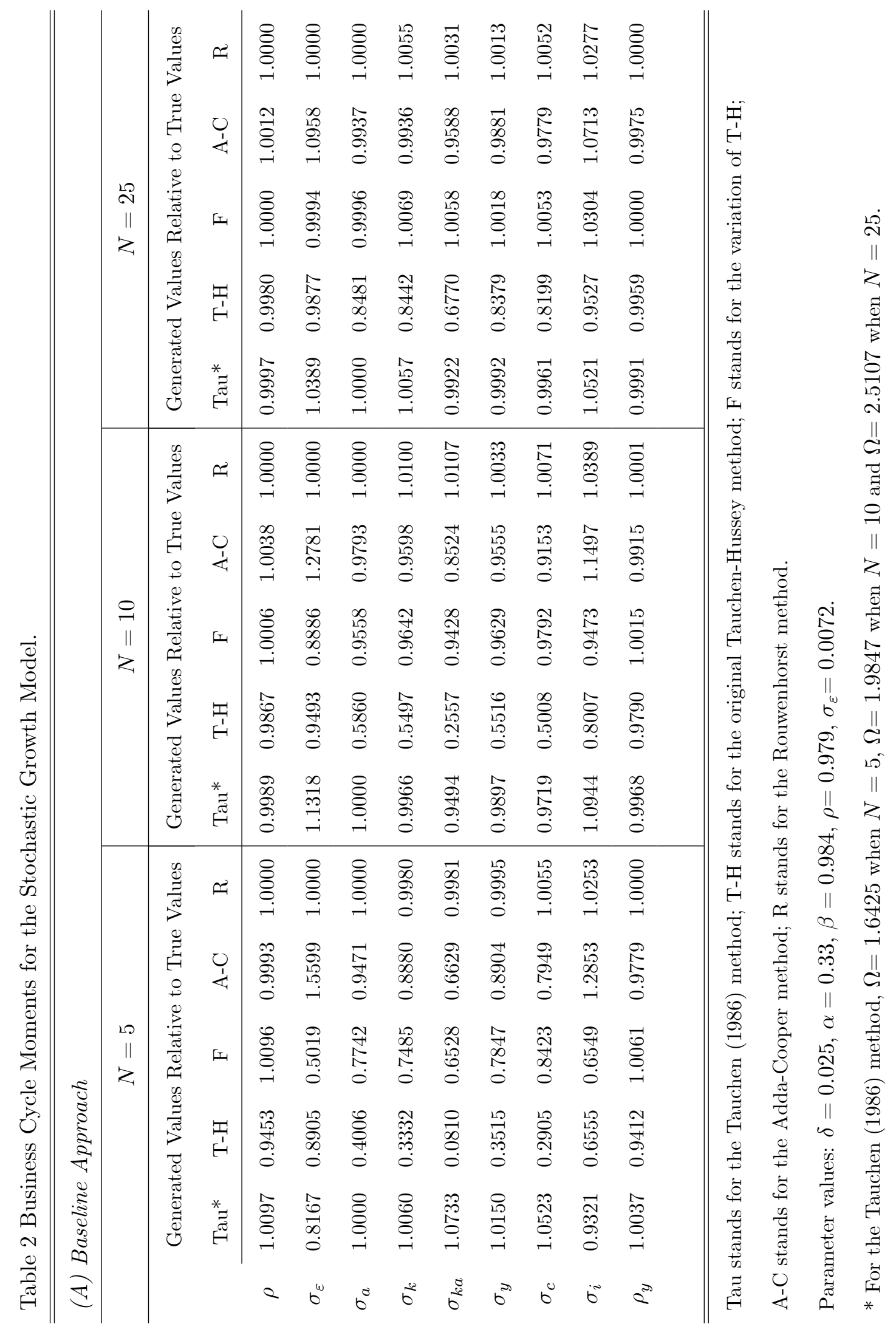




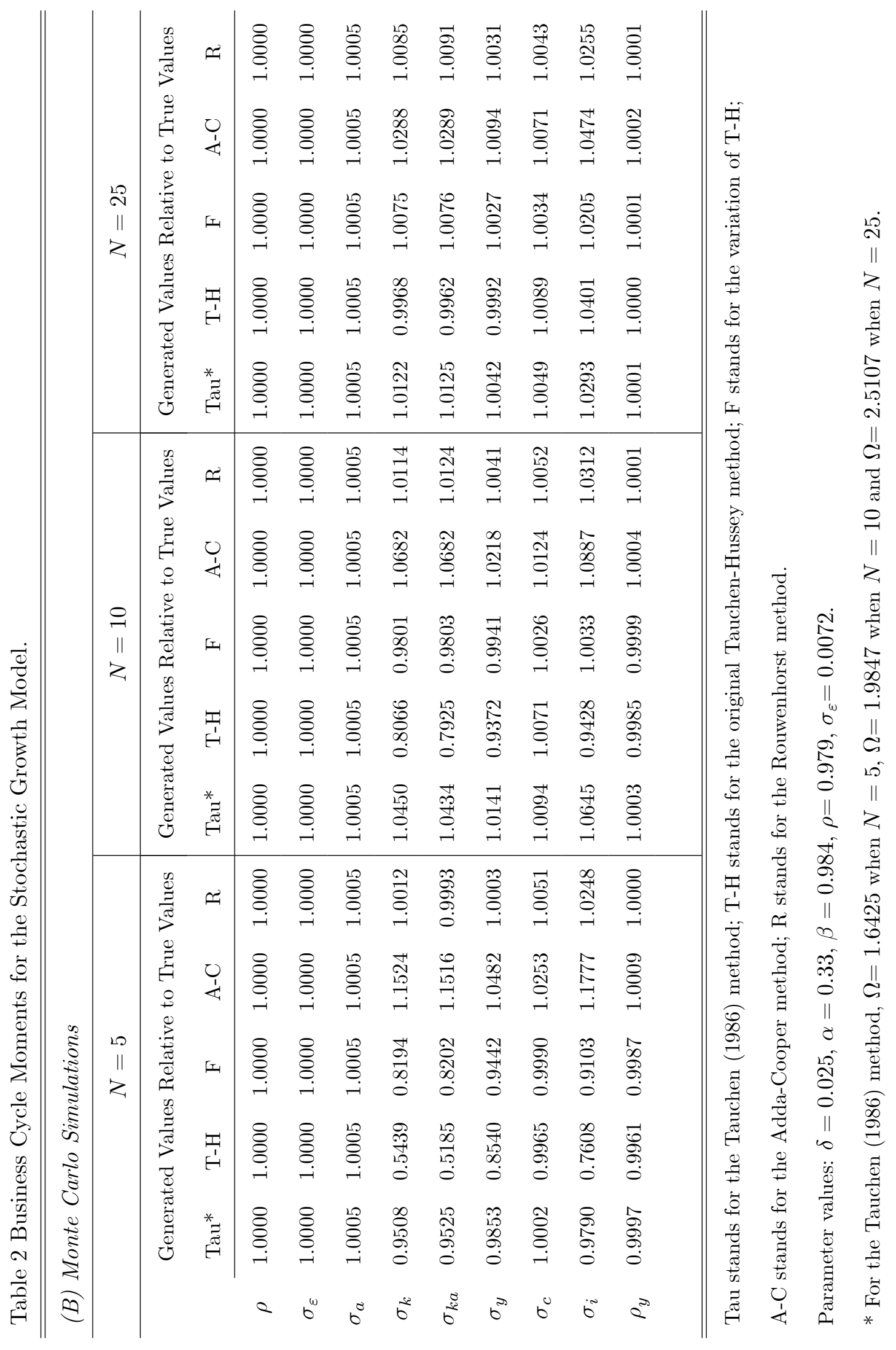




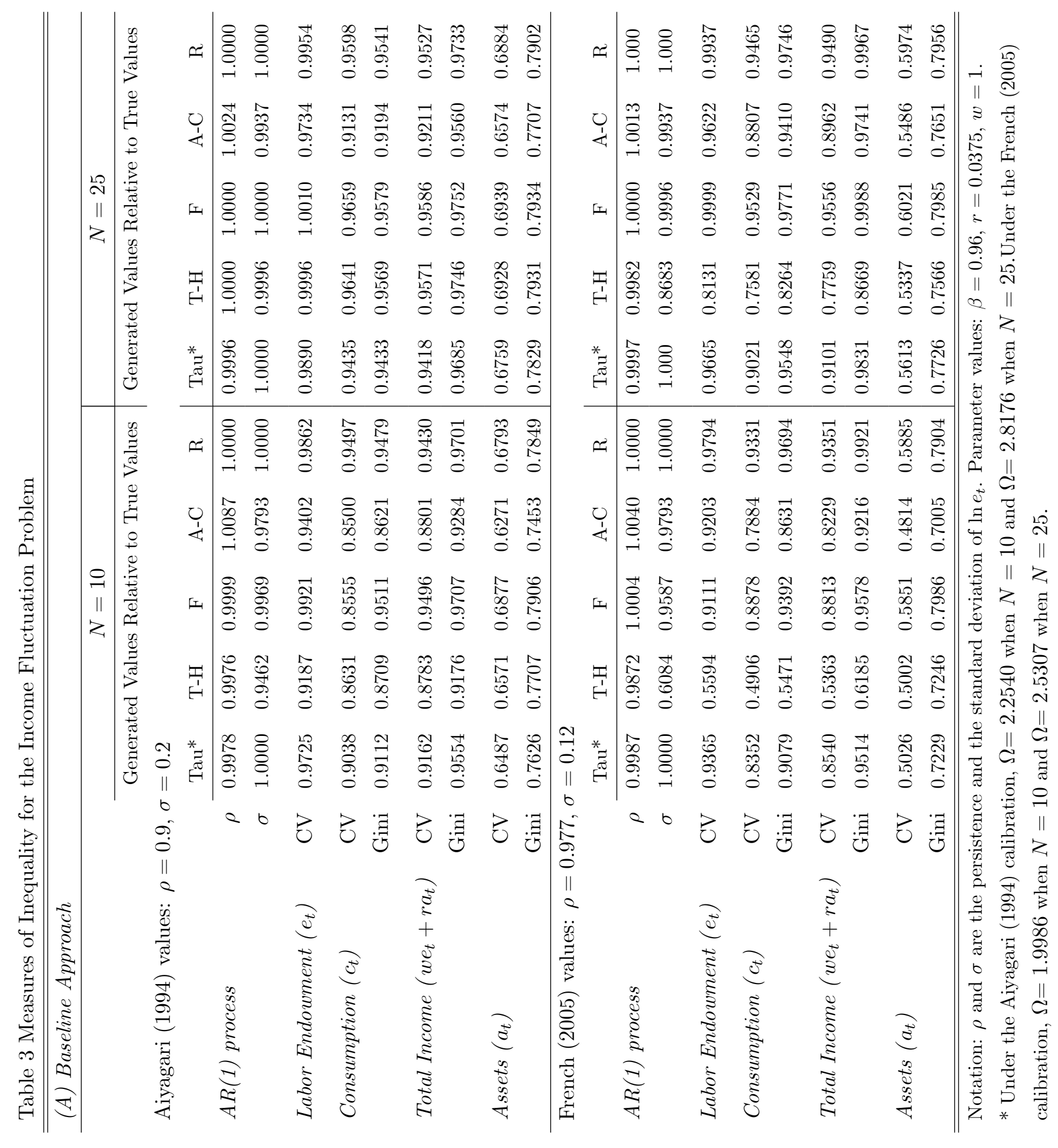




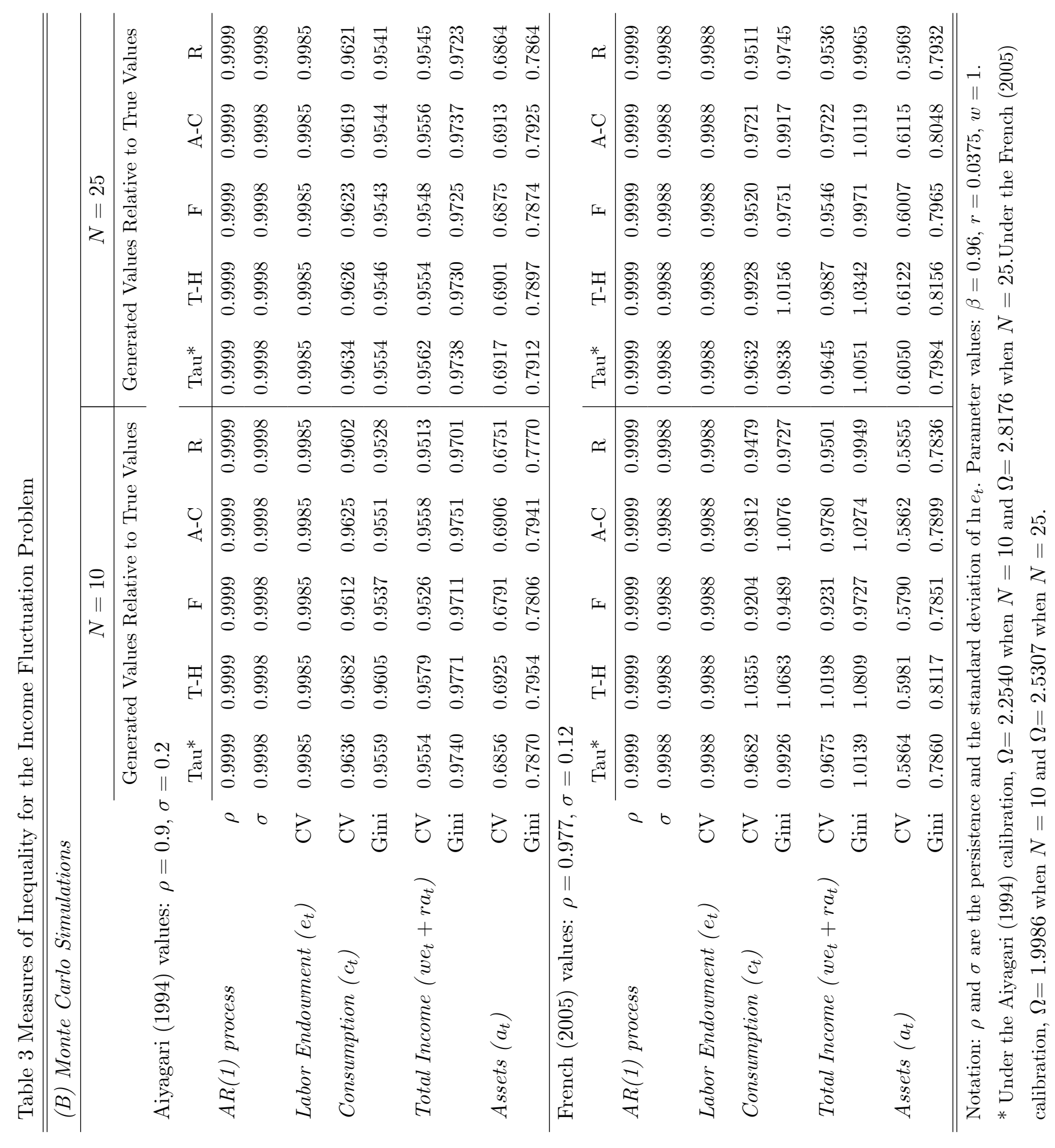




\section{Appendix}

\section{Preliminaries}

In this section we derive a set of equations that are useful in the following proofs. First using the binomial formula, the elements in the first and the last rows of $\Pi_{N}$ can be expressed as

$$
\pi_{1, j}^{(N)}=\left(\begin{array}{c}
N-1 \\
j-1
\end{array}\right) p^{N-j}(1-p)^{j-1}
$$

and

$$
\pi_{N, j}^{(N)}=\left(\begin{array}{c}
N-1 \\
j-1
\end{array}\right)(1-q)^{N-j} q^{j-1}
$$

for $j=1,2, \ldots, N$. For all other rows, the elements in $\Pi_{N}$ can be defined recursively using the elements in $\Pi_{N-1}$. Begin with the system for $N-1 \geq 2$. The system of polynomials is given by

$$
\Phi(t ; N-1, i)=[p+(1-p) t]^{N-1-i}(1-q+q t)^{i-1}=\sum_{j=1}^{N-1} \pi_{i, j}^{(N-1)} t^{j-1}
$$

for $i=1, \ldots, N-1$. There are two ways to relate this system to the one for $N$ :

$$
\Phi(t ; N, i)=[p+(1-p) t] \Phi(t ; N-1, i),
$$

for $i=1, \ldots, N-1$, and

$$
\Phi(t ; N, i)=(1-q+q t) \Phi(t ; N-1, i-1),
$$

for $i=2, \ldots, N$. Substituting (3) into (12) and rearranging terms gives

$$
\sum_{j=1}^{N} \pi_{i, j}^{(N)} t^{j-1}=\sum_{j=1}^{N-1} p \pi_{i, j}^{(N-1)} t^{j-1}+\sum_{j=1}^{N-1}(1-p) \pi_{i, j}^{(N-1)} t^{j}
$$

for $i=1, \ldots, N-1$. Similarly, substituting (3) into (13) would give

$$
\sum_{j=1}^{N} \pi_{i, j}^{(N)} t^{j-1}=\sum_{j=1}^{N-1}(1-q) \pi_{(i-1), j}^{(N-1)} t^{j-1}+\sum_{j=1}^{N-1} q \pi_{(i-1), j}^{(N-1)} t^{j}
$$

for $i=2, \ldots, N$. The following can be obtained by comparing the coefficients for $i=1,2, \ldots, N-1$,

$$
\pi_{i, 1}^{(N)}=p \pi_{i, 1}^{(N-1)}=(1-q) \pi_{(i-1), 1}^{(N-1)}
$$




$$
\pi_{i, j}^{(N)}=p \pi_{i, j}^{(N-1)}+(1-p) \pi_{i,(j-1)}^{(N-1)}=(1-q) \pi_{(i-1), j}^{(N)}+q \pi_{(i-1),(j-1)}^{(N)},
$$

for $j=2, \ldots, N-1$, and

$$
\pi_{i, N}^{(N)}=(1-p) \pi_{i,(N-1)}^{(N-1)}=q \pi_{(i-1), N}^{(N-1)} .
$$

\section{Proof of Proposition 1}

For any $N \geq 2$, the elements in the Rouwenhorst matrix $\Theta_{N}=\left[\theta_{i, j}^{(N)}\right]$ are governed by the following equations: For the elements in the first row,

$$
\theta_{1, j}^{(N)}= \begin{cases}p \theta_{1, j}^{(N-1)}, & \text { if } j=1, \\ p \theta_{1, j}^{(N-1)}+(1-p) \theta_{1,(j-1)}^{(N-1)}, & \text { if } j=2, \ldots, N-1, \\ (1-p) \theta_{1,(j-1)}^{(N-1)}, & \text { if } j=N .\end{cases}
$$

For the elements in the final row,

$$
\theta_{N, j}^{(N)}= \begin{cases}(1-q) \theta_{(N-1), j}^{(N-1)}, & \text { if } j=1, \\ (1-q) \theta_{(N-1), j}^{(N-1)}+q \theta_{(N-1),(j-1)}^{(N-1)}, & \text { if } j=2, \ldots, N-1, \\ q \theta_{(N-1),(j-1)}^{(N-1)}, & \text { if } j=N .\end{cases}
$$

For the elements in row $i=2, \ldots, N-1$,

$$
\theta_{i, j}^{(N)}= \begin{cases}\frac{1}{2}\left[p \theta_{i, j}^{(N-1)}+(1-q) \theta_{(i-1), j}^{(N-1)}\right], & \text { if } j=1, \\ \frac{1}{2}\left[(1-p) \theta_{i,(j-1)}^{(N-1)}+q \theta_{(i-1),(j-1)}^{(N-1)}\right], & \text { if } j=N,\end{cases}
$$

and for $j=2, \ldots, N-1$,

$$
\theta_{i, j}^{(N)}=\frac{1}{2}\left[p \theta_{i, j}^{(N-1)}+(1-p) \theta_{i,(j-1)}^{(N-1)}+(1-q) \theta_{(i-1), j}^{(N-1)}+q \theta_{(i-1),(j-1)}^{(N-1)}\right]
$$

For any given $\Theta_{N-1}$, the system of equations (17)-(20) defines a unique $\Theta_{N}$. Similarly, for any given $\Pi_{N-1}$, the system of equations (10)-(16) defines a unique $\Pi_{N}$. Since $\Theta_{2}=\Pi_{2}$, it suffices to show that the system (10)-(16) coincides with the system (17)-(20).

Consider the first row (i.e., $i=1)$ in $\Pi_{N}$. According to $(10), \pi_{1,1}^{(N)}=p \pi_{1,1}^{(N-1)}$, and $\pi_{1, N}^{(N)}=(1-p) \pi_{1,(N-1)}^{(N-1)}$. For $j=2, \ldots, N-1$, since

$$
\pi_{1, j}^{(N-1)}=\left(\begin{array}{c}
N-2 \\
j-1
\end{array}\right) p^{N-1-j}(1-p)^{j-1}
$$


and

$$
\left(\begin{array}{c}
N-1 \\
j-1
\end{array}\right)=\left(\begin{array}{c}
N-2 \\
j-1
\end{array}\right)+\left(\begin{array}{c}
N-2 \\
j-2
\end{array}\right)
$$

we have

$$
\pi_{1, j}^{(N)}=p \pi_{1, j}^{(N-1)}+(1-p) \pi_{1,(j-1)}^{(N-1)}
$$

This shows that the elements in the first row of $\Pi_{N}$ satisfy (17). Using (11) and the same procedure, one can show that the elements in the last row of $\Pi_{N}$ satisfy (18). The rest of the proof follows immediately from (14)-(16). For instance, for any row $i=2, \ldots, N-1$ in $\Pi_{N},(14)$ implies

$$
\pi_{i, 1}^{(N)}=\frac{1}{2}\left[p \pi_{i, 1}^{(N-1)}+(1-q) \pi_{(i-1), 1}^{(N-1)}\right]
$$

This coincides with the first equation in (19). Similarly, (15) and (16) can be used to derive the remaining equations in (19) and (20). Thus all the elements in row $i=2, \ldots, N-1$ in $\Pi_{N}$ satisfies (19) and (20). This completes the proof.

\section{Proof of Proposition 3}

As mentioned in the proof of Proposition 1, the first column of $\Pi_{N}$ is given by

$$
\pi_{i, 1}^{(N)}=p^{N-i}(1-q)^{i-1}
$$

for $i=1,2, \ldots, N$. Define $\widehat{\lambda}_{i}^{(N)}$ as in (4). Then

$$
\sum_{i=1}^{N} \widehat{\lambda}_{i}^{(N)} \pi_{i, 1}^{(N)}=[s p+(1-s)(1-q)]^{N}=s^{N}=\widehat{\lambda}_{1}^{(N)}
$$

For all other columns except the first one, an induction argument is used. First we know that the guess is correct when $N=2$. Suppose the guess is correct for some $N \geq 2$, i.e.,

$$
\widehat{\lambda}_{j}^{(N)}=\sum_{i=1}^{N} \widehat{\lambda}_{i}^{(N)} \pi_{i, j}^{(N)}, \quad \text { for } j=1,2, \ldots, N
$$


We have already proved that this is true when $j=1$, so proceed to $j=2, \ldots, N+1$. Using (4), the following can be derived

$$
\widehat{\lambda}_{i}^{(N+1)}= \begin{cases}s \widehat{\lambda}_{i}^{(N)}, & \text { for } i=1, \\ s \widehat{\lambda}_{i}^{(N)}+(1-s) \widehat{\lambda}_{i-1}^{(N)}, & \text { for } i=2, \ldots, N \\ (1-s) \widehat{\lambda}_{i-1}^{(N)}, & \text { for } i=N+1 .\end{cases}
$$

Using these one can obtain

$$
\sum_{i=1}^{N+1} \widehat{\lambda}_{i}^{(N+1)} \pi_{i, j}^{(N+1)}=\sum_{i=1}^{N} s \widehat{\lambda}_{i}^{(N)} \pi_{i, j}^{(N+1)}+\sum_{i=1}^{N}(1-s) \widehat{\lambda}_{i}^{(N)} \pi_{(i+1), j}^{(N+1)}
$$

A more detailed derivation of this result can be found in Kopecky and Suen (2009). Based on (15), the following can be obtained

$$
\pi_{i, j}^{(N+1)}=p \pi_{i, j}^{(N)}+(1-p) \pi_{i, j-1}^{(N)},
$$

and

$$
\pi_{i+1, j}^{(N+1)}=(1-q) \pi_{i, j}^{(N)}+q \pi_{i,(j-1)}^{(N)},
$$

for $j=2,3, \ldots, N$. Substituting these into (23) and rearranging terms gives

$$
\begin{aligned}
& \sum_{i=1}^{N+1} \widehat{\lambda}_{i}^{(N+1)} \pi_{i, j}^{(N+1)} \\
= & {[s p+(1-s)(1-q)] \sum_{i=1}^{N} \widehat{\lambda}_{i}^{(N)} \pi_{i, j}^{(N)}+[s(1-p)+(1-s) q] \sum_{i=1}^{N} \widehat{\lambda}_{i}^{(N)} \pi_{i,(j-1)}^{(N)} . }
\end{aligned}
$$

Using the induction hypothesis (21) and (22) gives,

$$
\sum_{i=1}^{N+1} \widehat{\lambda}_{i}^{(N+1)} \pi_{i, j}^{(N+1)}=s \widehat{\lambda}_{j}^{(N)}+(1-s) \widehat{\lambda}_{j-1}^{(N)}=\widehat{\lambda}_{j}^{(N+1)}
$$

for $j=2,3, \ldots, N$. Since $\sum_{i=1}^{N+1} \widehat{\lambda}_{i}^{(N+1)}=1$ and $\sum_{j=1}^{N+1} \pi_{i, j}^{(N+1)}=1$, the remaining equation for $j=N+1$ must be satisfied. This completes the proof. 


\section{References}

[1] Adda, J., Cooper, R., 2003. Dynamic Economics: Quantitative Methods and Applications. MIT Press, Cambridge, MA.

[2] Aiyagari, S.R., 1994. Uninsured Idiosyncratic Risk and Aggregate Saving. Quarterly Journal of Economics 109, 659-684.

[3] Aruoba, S., Fernández-Villaverde, J., Rubio-Ramírez, J., 2006. Comparing Solution Methods for Dynamic Equilibrium Economies. Journal of Economic Dynamics and Control 30, 2477-2508.

[4] Burnside, C., 1999. Discrete State-Space Methods for the Study of Dynamic Economies. In: Marimon, R., Scott, A. (Ed.), Computational Methods for the Study of Dynamic Economies. Oxford University Press, Oxford, 95-113.

[5] Chang, Y., Kim, S., 2006. From Individual to Aggregate Labor Supply: A Quantitative Analysis Based on a Heterogeneous Agent Economy. International Economic Review 47, 1-27.

[6] Chang, Y., Kim, S., 2007. Heterogeneity and Aggregation: Implications for Labor-Market Fluctuations. American Economic Review 97, 1939-1956.

[7] Christiano, L., Fisher, J., 2000. Algorithms for Solving Dynamic Models with Occasionally Binding Constraints. Journal of Economic Dynamics and Control 24, 1179-1232.

[8] den Haan, W.J., 2010. Comparison of Solutions to the Incomplete Markets Model with Aggregate Uncertainty. Journal of Economic Dynamics and Control 34, 4-27.

[9] Flodén, M., 2008a. A Note on the Accuracy of Markov-chain Approximations to Highly Persistent AR(1) Processes. Economics Letters 99, 516-520.

[10] Flodén, M., 2008b. Aggregate Savings When Individual Income Varies. Review of Economic Dynamics $11,70-82$.

[11] French, E., 2005. The Effects of Health, Wealth, and Wages on Labor Supply and Retirement Behaviour. Review of Economic Studies 72, 395-427.

[12] King, R.G., Rebelo, S.T., 1999. Resuscitating Real Business Cycles. In: Taylor, B.J., Woodford, M. (Ed.), Handbook of Macroeconomics, Volume 1. Elsevier, Amsterdam, 927-1007. 
[13] Kopecky, K.A., Suen, R., 2009. Finite State Markov-chain Approximations to Highly Persistent Processes. Working paper version.

[14] Krusell, P., Mukoyama, T., Şahin, A., Smith, A.A., 2009. Revisiting the Welfare Effects of Eliminating Business Cycles. Review of Economic Dynamics 12, 393-404.

[15] Krusell, P., Smith, A.A., 1998. Income and Wealth Heterogeneity in the Macroeconomy. Journal of Political Economy 106, 867-896.

[16] Lkhagvasuren, D., Galindev, R., 2008. Discretization of Highly-Persistent Correlated AR(1) Shocks. Unpublished manuscript, Concordia University.

[17] Pijoan-Mas, J., 2006. Precautionary Savings or Working Longer Hours? Review of Economic Dynamics $9,326-352$.

[18] Rouwenhorst, K.G., 1995. Asset Pricing Implications of Equilibrium Business Cycle Models. In: Cooley, T.F. (Ed.), Frontiers of Business Cycle Research. Princeton University Press, Princeton, NJ, 294-330.

[19] Santos, M.S., Peralta-Alva, A., 2005. Accuracy of Simulations for Stochastic Dynamic Models. Econometrica 73, 1939-1976.

[20] Storesletten, K., Telmer, C., Yaron, A., 1999. The Risk-Sharing Implications of Alternative Social Security Arrangements. Carnegie-Rochester Conference Series on Public Policy 50, 213-259.

[21] Tauchen, G., 1986. Finite State Markov-chain Approximations to Univariate and Vector Autoregressions. Economics Letters 20, 177-181.

[22] Tauchen, G., 1990. Solving the Stochastic Growth Model by using Quadrature Methods and ValueFunction Iterations. Journal of Business and Economic Statistics 8, 49-51.

[23] Tauchen, G., Hussey, R., 1991. Quadrature-Based Methods for Obtaining Approximate Solutions to Nonlinear Asset Pricing Models. Econometrica 59, 371-396.

[24] Taylor, J., Uhlig, H., 1990. Solving Nonlinear Stochastic Growth Models: A Comparison of Alternative Solution Methods. Journal of Business and Economic Statistics 8, 1-18. 